\title{
Micro and nanoengineering advances for the development and commercialization of Organ-on-chips
}

\author{
Adrian Martinez-Rivas ${ }^{1 *}$ Génesis K. González-Quijano \\ ${ }^{1}$ CIC, Instituto Politécnico Nacional (IPN). Av. Juan de Dios Bátiz, Esq. Miguel Othón de Mendizábal S/N, Nueva Industrial Vallejo, 07738, Ciudad de México \\ ${ }^{2}$ CONACYT-CNMN, IPN, Av. Luis Enrique Erro s/n, Nueva Industrial Vallejo, 07738, Ciudad de México
}

Advances in micro and nanoengineering have permitted to implement Organ-on-chips (OOC) that mimic accurately physiological functions. OOC are multichannel 3D microfluidic cell culture biodevices that mimic and model physiological mechanisms, activities, functions and responses of organs and tissues. These biomimetic microengineering approaches have gained significant relevance in biomedicine because they allow providing a better approximation of structure, behavior of cells and mechanisms of diseases to propose better diagnostic methods and personalized therapies [1].

OOC have been taken advantage of the semiconductor industry, like the case of Biomedical Microelectromechanical Systems (BioMEMS), because of the related semiconductor materials and the well-established micro and nanofabrication processes. Some techniques commonly employed are photolithography to create molds made of silicon, quartz or plastic, and wet or dry etching for micromachining these structures. Therefore, some modern micro and nanofabrication techniques such as soft-lithography and microcontact printing have also been used. These latter techniques employ the so-called poly(dimethylsiloxane) (PDMS) as it has advantages for 3D culture such as gas permeability [2] but it also has some drawbacks such as adsorbing small hydrophobic molecules [3].

One interesting microengineering work using PDMS consisted in the development of an OCC called lung-on-chips that mimics the alveolar-capillary interface of the human lung, to study the cytotoxicity of silica nanoparticles [4]. These with microfabricated pores that permitted it to have an air-liquid interface. However, one limitation has been that these artificial membranes are thicker than the interstitium between the alveolar epithelium and capillary endothelium in vivo (1 $\mu \mathrm{m}$ in thickness) [5]. Hence, the ultrathin porous membrane issue, which would mimic the in vivo condition, has to be overcome with novel materials and microengineering methodologies. Then, in a very recent work authors proposed the use and commercialization of $100-300 \mathrm{~nm}$ in vitro barriers membranes [6], which are comparable to the physiological separation distances in tissue layers and permit the transfer of cytoplasmic cargo between the co-cultured cells, being optically transparent for the fluorescence and phase image of the cells, positioned on both sizes of it. Those ultrathin membranes were made of tetraethyl-orthosilicate (TEOS)-derived $\mathrm{SiO} 2$ via plasma-enhanced chemical vapor deposition (PE-CVD) and the stress of the membrane was controlled by modifying the deposition parameters.

In this context, micro and nanoengineering methodologies have permitted the OOC include biosensors, with some recent advances related to the measurement of transepithelial electrical resistance (TEER), aiming to study the Blood-brain barrier (BBB) that is associated with diseases such as Alzheimer, multiple sclerosis, stroke, cancer, and vascular malformations [7]. Furthermore, some extraordinary nanomaterial as graphene are being used for live cell biosensing [8]. The development and integration of biosensors for studying cell-cell interactions and intracellular cell process will grow in near future.

On the other hand, rapid prototyping is needed to develop highthroughput microfluidic platforms as part of OOC and put them into the market. In this regard, the use of $3 \mathrm{D}$ bioprinting techniques such as stereolithography (SLA), Fused Deposition Modeling (FDM), Electron Beam Melting (EBM), Bioprinter and Inkjet Printing are impacting $[9 ; 10 ; 11]$ and recently a liver-on-a-chip that used gelatin hydrogel to study protein adsorption and drug metabolism was elaborated by $3 \mathrm{D}$ bioprinting, in only one step [12].

The OCC have several advantages to study medical conditions invitro, permitting to receive drugs approvals more rapidly compared to the difficulty and the cost that represents to perform clinical studies in vivo, which has motivated some companies such as TissUse GmbH, to design Multi-Organ-Chip Platforms, having cell compartments to include different types of cells [13]. Governmental programs mainly from USA and Europe are founding projects related to OCC and large companies are are investing in the development and commercialization of this technology [14]. One commercial product is the liverchip device, developed by C.N. Bioinnovations, it includes touchscreen controller and dynamic perfusion micro-pumps [15]. IThe development and utilization of OOC will have an enormous impact next year both in technology and economy worldwide.

\section{Acknowledgments}

AMR would like to thank the financial support of SIP-IPN through the projects 20160181 and 20170067. GKGQ thanks to CONACYT and CNMN-IPN for the support to develop her research activities.

\section{References}

1. Bhatia SN, Ingber DE2 (2014) Microfluidic organs-on-chips. Nat Biotechnol 32: 760772. [Crossref]

2. Halldorsson S, Lucumi E, Gómez-Sjöberg R, Fleming RMT (2015) Advantages and challenges of microfluidic cell culture in polydimethylsiloxane devices. Biosens Bioelectron 63: 218-231.

Correspondence to: Adrian Martinez-Rivas, CIC, Instituto Politécnico Nacional (IPN). Av. Juan de Dios Bátiz, Esq. Miguel Othón de Mendizábal S/N, Nueva Industrial Vallejo, 07738, Ciudad de México, E-mail: amartinezri@cic.ipn.mx

Key words: Micro and nanofabrication, Organ-on-chips, Biomedicine, Microfluidic devices, Micro and nanoengineering

Received: May 21, 2017; Accepted: June 06, 2017; Published: June 08, 2017 
3. Toepke MW, Beebe DJ (2006) PDMS absorption of small molecules and consequences in microfluidic applications. Lab Chip 6: 1484-1486. [Crossref]

4. Huh D, Matthews BD, Mammoto A, Montoya-Zavala M, Hsin HY, et al. (2010) Reconstituting organ-level lung functions on a chip. Science 328: 1662-1668. [Crossref]

5. Huh D, Kim HJ, Fraser JP, Shea DE, Khan M, et al. (2013) Microfabrication of human organs-on-chips. Nat Protoc 8: 2135-2157. [Crossref]

6. Robert NC, Stephanie MC, Andrea RM, Jon-Paul SD, James AR et al. (2017) Ultrathin transparent membranes for cellular barrier and co-culture models. Biofabrication 9: 015019. [http://stacks.iop.org/1758-5090/9/i=1/a=015019]

7. van der Helm MW, Odijk M, Frimat J-P, van der Meer AD, Eijkel JCT et al. (2016) Direct quantification of transendothelial electrical resistance in organs-on-chips. Biosens Bioelectron 85: 924-929.

8. Kim T-H, Lee D, Choi J-W (2017) Live cell biosensing platforms using graphene-based hybrid nanomaterials. Biosens Bioelectron 94: 485-499.
9. Ho CMB, Ng SH, Li KHH, Yoon Y-J (2015) 3D printed microfluidics for biological applications. Lab Chip 15: 3627-3637. [http://dx.doi.org/10.1039/C5LC00685F].

10. Yang Q, Lian Q, Xu F (2017) Perspective: Fabrication of integrated organ-on-a-chip via bioprinting. Biomicrofluidics 11: 031301. [Crossref]

11. Au AK, Huynh W, Horowitz LF, Folch A (2016) 3D-Printed Microfluidics. Angew Chem Int Ed Engl 55: 3862-3881. [Crossref]

12. Lee H, Cho DW (2016) One-step fabrication of an organ-on-a-chip with spatial heterogeneity using a 3D bioprinting technology. Lab Chip 16: 2618-2625. [Crossref]

13. TissUse GmbH. https://www.tissuse.com/en/products/2-organ-chip/ (accessed May 17th, 2017).

14. Balijepalli A, Sivaramakrishan V (2017) Organs-on-chips: research and commercial perspectives. Drug Discov Today 22: 397-403. [Crossref]

15. cnBio innovations. http://cn-bio.com/products/ (accessed May 17th, 2017).

Copyright: (2017 Martinez-Rivas A. This is an open-access article distributed under the terms of the Creative Commons Attribution License, which permits unrestricted use, distribution, and reproduction in any medium, provided the original author and source are credited. 